\title{
Optimization Design and Structural Dynamic Analysis of Cycling
}

\author{
Zhuang Min* \\ Hangzhou Polytechnic, Zhejiang, Hangzhou 311402, China
}

\begin{abstract}
In this paper, the research object is the cycling equipment which is a common fitness equipment. The dynamic characteristics of the cycling equipment were optimized in a virtual prototype, and analysis was carried out on its structural mechanical characteristics and dynamic performance, etc. The dynamic characteristics of the cycling equipment were obtained through modal, random response and harmonic response analyses, and the structural reasonability after optimization was verified. This research will ptovide clues to avoid unreasonable design of the cycling equipment, besides an effective analysis was made and assessment method for cycling equipment design was proposed.
\end{abstract}

Keywords: Dynamics, finite element, optimization of cycling equipment.

\section{INTRODUCTION}

With the improvement of people's livelihood, fitness industry enjoys rapid development, so a stricter requirement is proposed for the innovation and $\mathrm{R} \& \mathrm{D}$ of fitness equipment. The researchers provide reasonable parameters according to ergonomics, and simulate it by means of the theories of kinematics of mechanism, thus optimizing the parameters of the cycling equipment, which can achieve the highest design efficiency. In this paper, simulation and optimization for the simplified mechanism of cycling equipment was realized by Adams software. After optimization, some of its mechanical characteristics were analyzed by building a finite element model of the cycling equipment. The finite element model was used for verifying that the cycling equipment of this dimension could meet the requirement of intensity in daily use. The results show that the speed stationarity of the optimized cycling equipment is improved within its operating stroke, which improves the comfort. The optimization of the mechanism design is realized by means of the optimization design of many-body dynamics software and finite element software, which provides a feasible design method for cycling equipment.

\section{DYNAMICAL MODEL OF CYCLING EQUIPMENT}

\subsection{Dimensional Design of Cycling Equipment Based on Ergonomics}

With cycling equipment, people can achieve the goal of exercise by sitting on the seat with the hands holding the handles, the feet pedaling on the pedals and the body keeping straight and upright, bending and stretching the arms repeatedly and moving the pedals. The basic design requirements of its movement function include: (1) the structure

*Address correspondence to this author at the Hangzhou Polytechnic, Zhejiang, Hangzhou311402, China; Tel: +86 -571-56700055;

E-mail: zhuangmin163@163.com dimensions of the cycling equipment comply with the human dimensions of the exercisers. (2) the reasonable structural form and structure parameters are designed to ensure the comfortable sports inertia of the cycling equipment. (3) the movement load of the cycling equipment complies with principles of dynamics. (4) beautiful appearance complies with the design requirements such as athlete psychology, etc. [1]. To cater to the fitness demands of most people, the $95^{\text {th }}$ percentiles of male at the age group of 18-66 and the $5^{\text {th }}$ percentiles of female at the age group of 18-55 are selected as a basis for the upper and lower limits of the dimensions. The spatial position of the handles of the cycling equipment should be set within the functional dimension range of the exercisers' upper limbs. The distance between the exerciser and the handles should be not less than the minimum dimension of the $95^{\text {th }}$ percentiles of male forearm function and not larger than the maximum dimension of the $5^{\text {th }}$ percentiles of female forearm function.

\subsection{Dynamical Optimization Model of the Cycling Equipment}

The cycling equipment is mainly composed of base, seat and its connectors, handles and their connectors as well as connectors. The structural parts of the cycling equipment form a contra-quadrilateral mechanism, as shown in Fig. (1). To simplify the analysis, the friction force between the kinematic pair during motion is ignored.

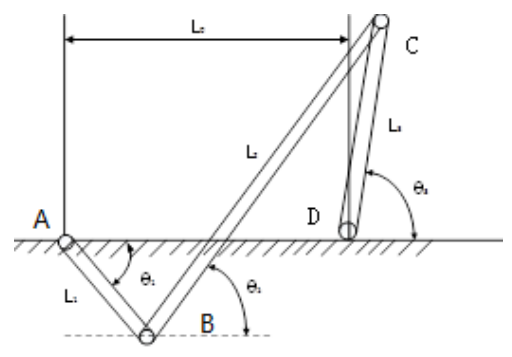

Fig. (1). Planar model of the cycling equipment. 
A rectangular coordinate system for the entire mechanism is built, with the hinge between the base and the handles as the origin, positive direction of $\mathrm{x}$ axle directing the hinge between the base and the seat. The coordinates of each vertex of the link mechanism are indicated by initial angle $\theta_{1}$ passing the handles.

Point A, $\mathrm{x}$ coordinate $0, \mathrm{y}$ coordinate 0

Point D, x coordinate $L_{0}$, y coordinate 0

Point B, x coordinate $L_{1} \cos \theta_{1}$, y coordinate $L_{1} \sin \theta_{1}$

Point C, x coordinate $L_{0}+L_{3} \cos \theta_{3}$, y coordinate $L_{3} \sin \theta_{3}$

Independent analysis was carried out on the bars, as shown in Fig. (2). The structural component bears binding force $F_{R i-1}$ at revolute pair i-1. Similarly, the structural component bears constraint reaction $F_{R i}$ at revolute pair $\mathrm{i}+1$.

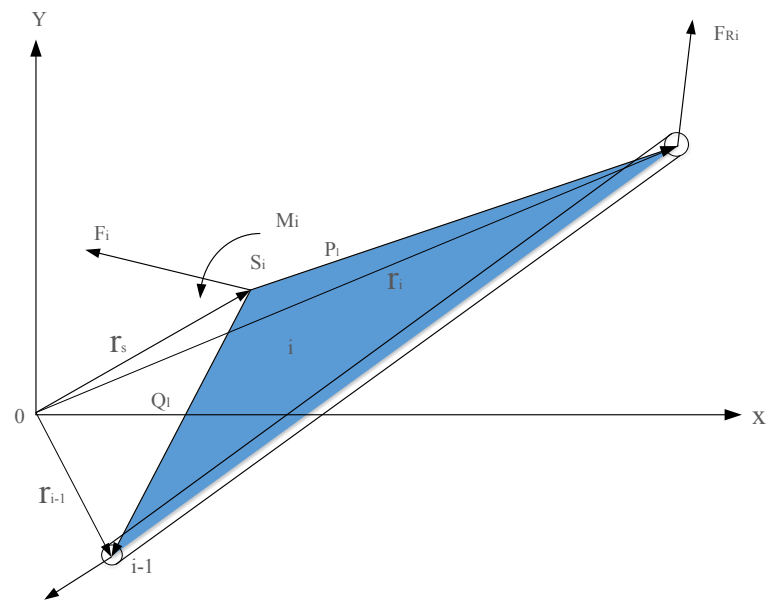

Fig. (2). Force analysis on bar i.

The following formulas can be acquired according to Alembert principle

$$
\left\{\begin{array}{l}
F_{R_{\mathrm{i}}}-F_{R_{i-1}}+F_{i}=m_{l} \dot{s}_{l} \\
p_{l} \times F_{R_{\mathrm{i}}}-q_{l} \times F_{R_{i-1}}+M_{i}=J_{i} \ddot{\phi}_{I}
\end{array}\right.
$$

Where $m_{i}$ is the mass of the structural components, $J_{i}$ is the rotational inertia of the structural components, $P_{l}$ and $Q_{l}$ are the vectors from the centroid to revolute pairi-1and i respectively, $\ddot{s_{i}}$ and $\ddot{\phi}$ are the linear acceleration and angular acceleration of the centroid respectively. The dynamical equations of rod $L_{1}, L_{2}$ and $L_{3}$ can be acquired. The equations are rewritten as follows by converting vectors into scalars:

$$
\left\{\begin{array}{l}
F_{R_{i} x}-F_{R_{i-1} x}=m_{i} x_{s i}-F_{i x} \\
F_{R_{i} y}-F_{R_{i-1} y}=m_{i} y_{s i}-F_{i x} \\
p_{i x} \times F_{R i y}-p_{i y} \times F_{R i x}-q_{i x} \times F_{R i-1 y} \\
+q_{i y} \times F_{R i-1 x}+M d=J_{i} \phi_{i}-M_{i}
\end{array}\right.
$$

Where $x_{s i}$ and $\ddot{y}_{s i}$ are the components of the acceleration of the centroid of the structural components on the two axles, $p_{i x}$ and $p_{i v}$ are the components of the vectors of the structural components on $\mathrm{x}$ axle and y axle respectively. Similarly, the nine equilibrium equations of $\operatorname{rod} L_{1}, L_{2}$ and $L_{3}$ can be acquired, thus forming system of linear equations $A R=B_{1}-B \cdot$ A in the following equation is a known as $9 \times 9$ square matrix as follows:

$$
A=\left[\begin{array}{cccccccccc}
-1 & & 1 & & & & & & \\
& -1 & & 1 & & & & & \\
q_{1 y} & q_{1 x} & -p_{1 y} & -p_{1 x} & & & & & \\
& & -1 & & 1 & & & & \\
& & & -1 & & & & & \\
& & q_{2 y} & -q_{2 x} & -p_{2 y} & p_{2 x} & & & \\
& & & & -1 & & 1 & & \\
& & & & & -1 & & 1 & \\
& & & & & q_{3 y} & -q_{3 x} & -p_{3 y} & p_{3 x}
\end{array}\right]
$$

In matrix $\mathrm{A}$, the elements are related to the positions at the centroid of the structural components. $\mathrm{B}$ is a known 9-dimensional array, as shown in the following equation:

$$
\begin{aligned}
& B=\left[\begin{array}{llll}
F_{1 x} & F_{1 y} & M_{1} & F_{2 x}
\end{array}\right. \\
& \left.\begin{array}{lllll}
F_{2 y} & M_{2} & F_{3 x} & F_{3 y} & M_{3}{ }^{T}
\end{array}\right]
\end{aligned}
$$

$B_{1}$ is a known 9-dimensional array which includes all the forces and moments of the mechanism:

$$
\begin{aligned}
& B_{1}=\left[\begin{array}{ccccc}
m_{1} \ddot{x}_{s 1} & m_{1} \ddot{y}_{s 1} & J_{1} \ddot{\phi} & m_{2} \ddot{x}_{s 2} \\
m_{2} \ddot{y}_{s 2} & J_{2} \ddot{\phi_{2}} & m_{3} \ddot{x}_{s 3} & m_{3} \ddot{y}_{s 3} & J_{2} \ddot{\phi_{2}}
\end{array}\right]^{T}
\end{aligned}
$$

$\mathrm{R}$ is an unknown 9-dimensional array to be solved:

$$
\begin{aligned}
& R=\left[\begin{array}{lllll}
F_{R_{1 x}} & F_{R_{1 y}} & F_{R_{2 x}} & F_{R_{2 y}} \\
F_{R_{3 x}} & F_{R_{3 y}} & F_{R_{4 x}} & F_{R_{4 y}} & M_{d}
\end{array}\right]^{T}
\end{aligned}
$$

In the array, $F_{R_{i x}}$ and $F_{R_{i j}}$ are the component forces along the rectangular coordinates of the kinematic pair of the structural component to be solved, and $M_{d}$ is driving moment. The aforesaid is the equilibrium equation of kinetostatic analysis of the cycling equipment. Analysis of kinematic 
parameters is required before dynamic analysis to obtain the displacements, velocities and accelerations of each part. Then the parameters are substituted in the aforesaid array to solve the counter-force and driving load on the kinematic pair.

Optimization design is carried out on the basis of parameterization of design variables. The objective design is optimized under the condition that the constraint conditions and the value range of the variables are met. In this paper, an objective function should be built after taking the rod length as the design variable. The cycling equipment requires fine drive characteristics during its operating stroke, with the minimum driving angle should be not less than $40-50^{\circ}$. In this model, the angle between connecting rods $\mathrm{AB}$ and $\mathrm{BC}$ as well as the angle between connecting rods $\mathrm{BC}$ and $\mathrm{CD}$ were measured by means of measure-angle. They were judged by judgment sentence IF. When the angle is larger than $180^{\circ}$, Temp1 $=180-\theta_{A B C}$, Temp2=180- $\theta_{B C D}$. According to international standard, the range for the upper limbs to stretch forward is $712-892 \mathrm{~mm}$, so $d_{F G} \leq 892$. In this paper, the four-bar linkage mechanism must meet the conditions of a closed plane four bar mechanism. Therefore, the following constraint conditions are required:

$g_{1}=\theta_{A B C} \geq 40^{\circ}$

$g_{2}=\theta_{B C D} \geq 40^{\circ}$

$g_{3}(z)=z_{1}+z_{2}-z_{3}-z_{4} \leq 0$

$g_{4}(z)=z_{1}+z_{3}-z_{2}-z_{4} \leq 0$

$g_{5}(z)=z_{1}+z_{4}-z_{2}-z_{3} \leq 0$

$g_{6}=d_{F G} \leq 892$

Meanwhile, the driving angle of the cycling equipment during operating stroke should be as large as possible, so as to ensure better driving performance and less requirement on the materials while other conditions are similar. The optimization objective should be the average values of $\theta_{A B C}$ and $\theta_{B C D}$ during the entire simulation process. However, the parameter optimization and simulation of Adams only allows single objective optimization, but the four-bar linkage mechanism is an optimization problem with multiple variables and multiple objectives. Therefore, the author needs to transform the multi-objective optimization problem into a single objective optimization problem, i.e.:

$$
\begin{aligned}
& \max F(\mathrm{x})=\mathrm{k}_{1}^{*} \max \left(F_{1}\right) \\
& +\mathrm{k}_{2} * \max \left(F_{2}\right)
\end{aligned}
$$

$\mathrm{k}_{1}$ and $\mathrm{k}_{2}$ are the weight values of the single objective optimization in the multi-objective optimization. The selection of the weight values can be changed according to design requirement. In this paper, they are 1 respectively, for the purpose of simplification.

\subsection{Simulation and Optimization for the Parameters of the Cycling Equipment}

In this paper, the reasonable parameters were acquired by constructing the simplified cycling equipment model. Then, simulation and optimization were carried out on the simplified mechanism of the cycling equipment by Adams software.

After building the design variables, constraint conditions and the objective function, the parameters were calculated and optimized during one working cycle in Simulate-Design Evaluation Tools. It was tedious to list all the coordinates affecting the rod length, so the variations of the coordinates of Point $\mathrm{C}$ during each iteration, were chosen as the reference. In Table 1, $\mathrm{x}$ coordinate of Point $\mathrm{C}$ oscillates before quickly convergence into the early iteration, while its y coordinate changes smoothly. This proves that the design of cycling equipment based on ergonomics is consistent with the optimized design to some extent.

Table 1. The variation trend of the objective function and the variations of the $x$ and $y$ coordinates of the parameterized Point 4 corresponding to it.

\begin{tabular}{|c|c|c|c|}
\hline $\begin{array}{c}\text { Number of } \\
\text { Iterations }\end{array}$ & $\begin{array}{c}\text { Value of the } \\
\text { Objective } \\
\text { Function }\end{array}$ & $\begin{array}{c}\boldsymbol{x} \text { value of Point } \\
\text { C }\end{array}$ & $\begin{array}{c}\boldsymbol{y} \text { value of Point } \\
\text { C }\end{array}$ \\
\hline \hline 1 & 160.02 & 58.278 & 44.987 \\
\hline 2 & 163.81 & 63.148 & 43.612 \\
\hline 3 & 166.25 & 62.035 & 43.270 \\
\hline 4 & 166.62 & 62.270 & 43.200 \\
\hline 5 & 166.69 & 61.266 & 43.200 \\
\hline 6 & 166.71 & 61.234 & 43.249 \\
\hline 7 & 166.74 & 60.561 & 43.703 \\
\hline 8 & 166.75 & 59.923 & 43.789 \\
\hline 9 & 166.75 & 60.136 & 43.679 \\
\hline 10 & 166.76 & 60.136 & 43.679 \\
\hline
\end{tabular}

Fig. (3) shows the variations of the forces on each revolute joint after optimization. Joints1, 2, 3 and 4 correspond to the revolute joints at $\mathrm{A}, \mathrm{B}, \mathrm{C}$ and $\mathrm{D}$ respectively, of the link mechanism of the cycling equipment. A comparison is carried out on the force-bearing condition on one of the revolute joints before and after optimization. The diagram shows that the force-bearing peak and average value of Point $\mathrm{D}$ which is the most typical are the maximum. Therefore, the revolute joint at Point D was selected for optimization. As shown in Fig. (4), Iter_1curves indicates the force-bearing curve after optimization; Iter_ 5 curve indicates the force-bearing curve before optimization. The difference between two curves is not obvious at the initial stage in one operating stroke. However, the difference value between the two increases as time goes by. After optimization, the average force-bearing value of the revolute joint in the operating stroke decreases, which is conducive to increasing the service life of the cycling equipment. 


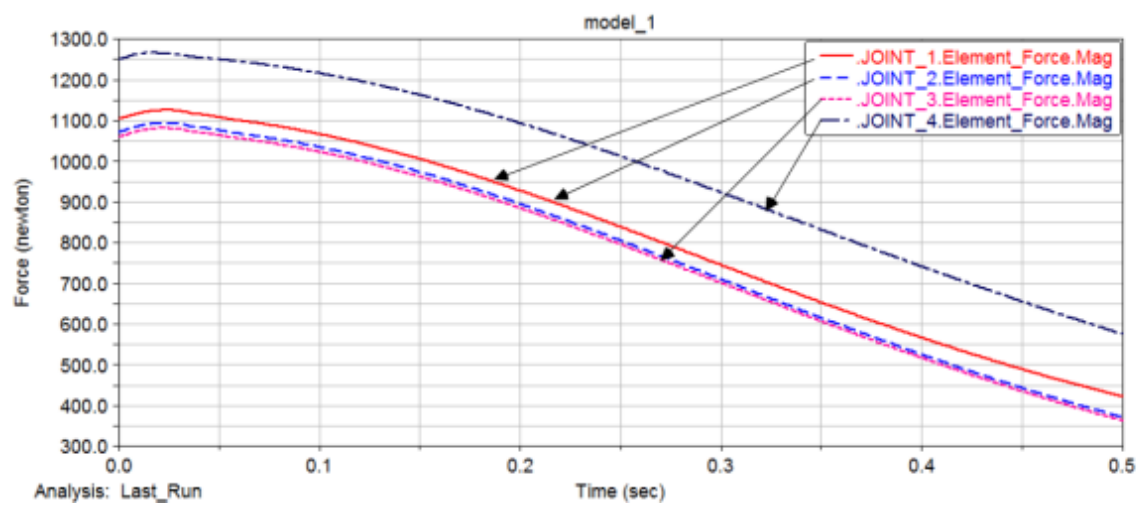

Fig. (3). Force-bearing curve of the revolute joints after optimization.

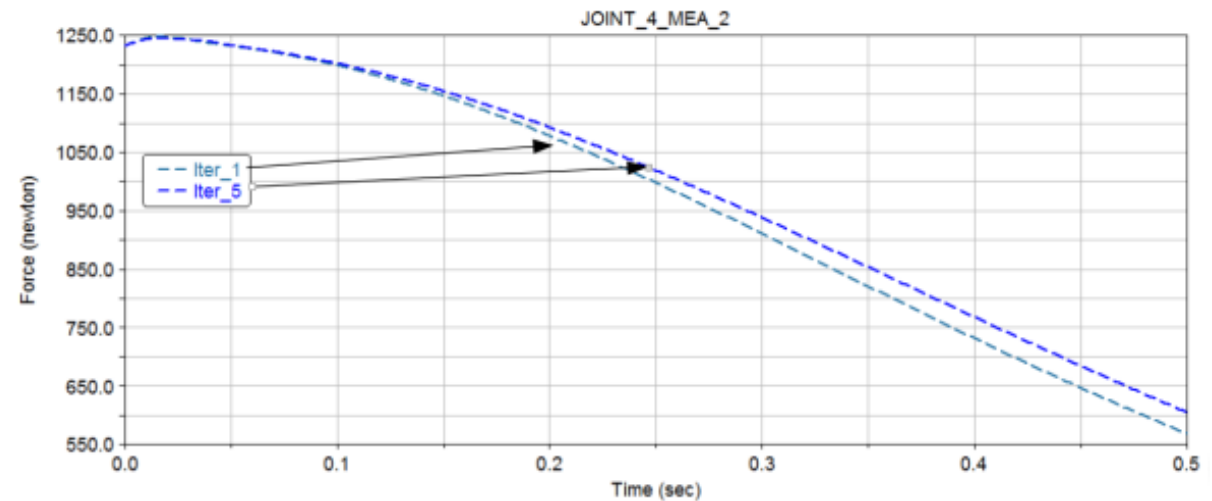

Fig. (4). Force-bearing variations of the revolute joint at point D before and after optimization.

\section{DYNAMIC ANALYSIS ON THE PARTS OF THE CYCLING EQUIPMENT AFTER OPTIMIZATION}

The base is an important constituent part of the cycling equipment. All the parts are installed on the base which bears all the loads when working. The deformation magnitude and dynamic characteristics of the base will directly affect the product precision and the service life of the cycling equipment. Reasonable design of the base is the premise and guarantee for sound static and dynamic performance. The base is made of Q345E, with the yield strength of $345 \mathrm{MPa}$, tensile strength of $600 \mathrm{MPa}$, elasticity modulus of $2.06 \mathrm{e} 5 \mathrm{M}$ PaandPoisson's ratio of 0.28 .

\subsection{Structural Dynamic Analysis on the Base of the Cy- cling Equipment}

The finite element model of the base was built by referencing the principle for mesh generation in References [2-5], as shown in Fig. (5):

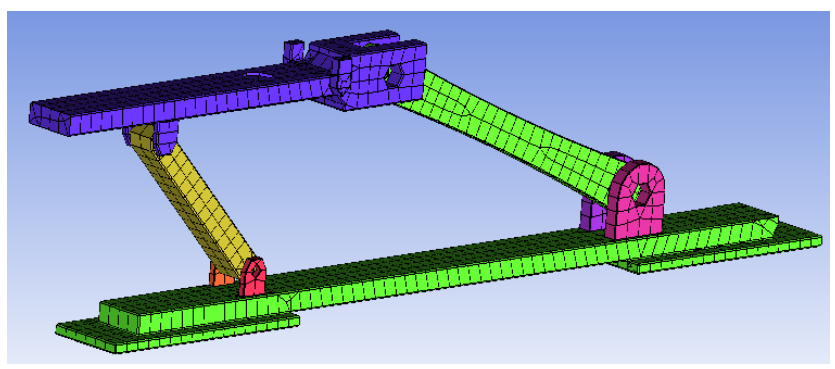

Fig. (5). Finite element model of the cycling equipment.
Fixed Support is used at the bottom to simulate the base which is fixed on the floor. Modal analysis is the basis for dynamic analysis and optimization for the cycling equipment structure. The first 6 orders of inherent frequencies of the base are acquired through calculation, as shown in Table 2:

Table 2. The first 6 orders of inherent frequencies of the base.

\begin{tabular}{|c|c|c|c|c|c|c|}
\hline Order & $\mathbf{1}$ & $\mathbf{2}$ & $\mathbf{3}$ & $\mathbf{4}$ & $\mathbf{5}$ & $\mathbf{6}$ \\
\hline \hline Frequency & 49.259 & 136.3 & 145.2 & 408.19 & 441.89 & 479.48 \\
\hline
\end{tabular}

According to Table 2, the frequency distribution of the base is as follows: the first frequency band includes the first 3 orders of modalities which are in the range of $49 \sim 145 \mathrm{~Hz}$, of which the vibration mode is the swinging of the upper part of the base in $\mathrm{Y}$ direction. A typical vibration mode of the $1^{\text {st }}$ order of modality is as shown in Fig. (6). The second frequency band includes the $4-6^{\text {th }}$ orders of modalities, which are in the range of 408-561. The frequency difference among the $4-6^{\text {th }}$ orders of modalities is small, when the vibration mode is the torsion of the base in three directions. A typical vibration mode of the 5 th order of modality is as shown in Fig. (7). 


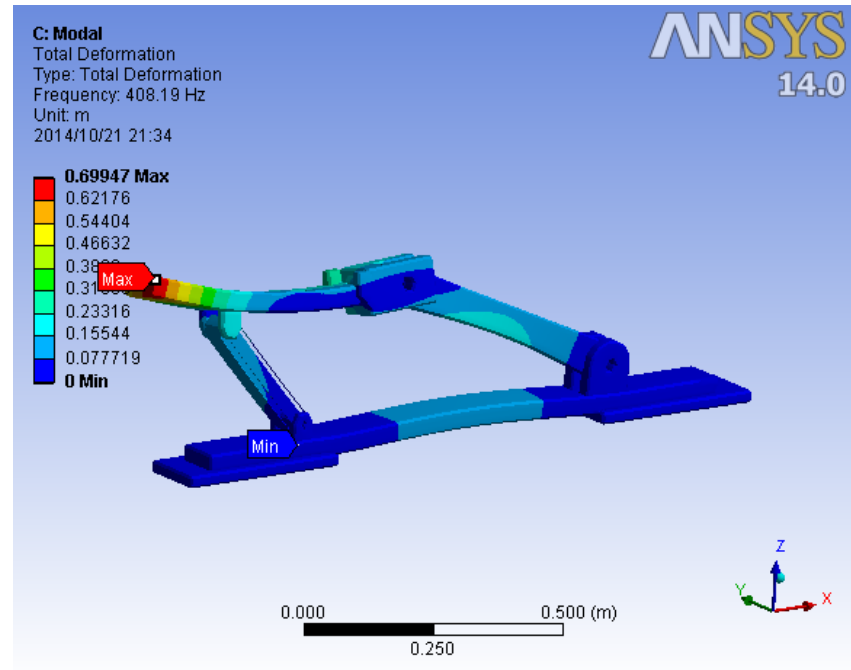

Fig. (6). The $4^{\text {th }}$ order of modality of the base.

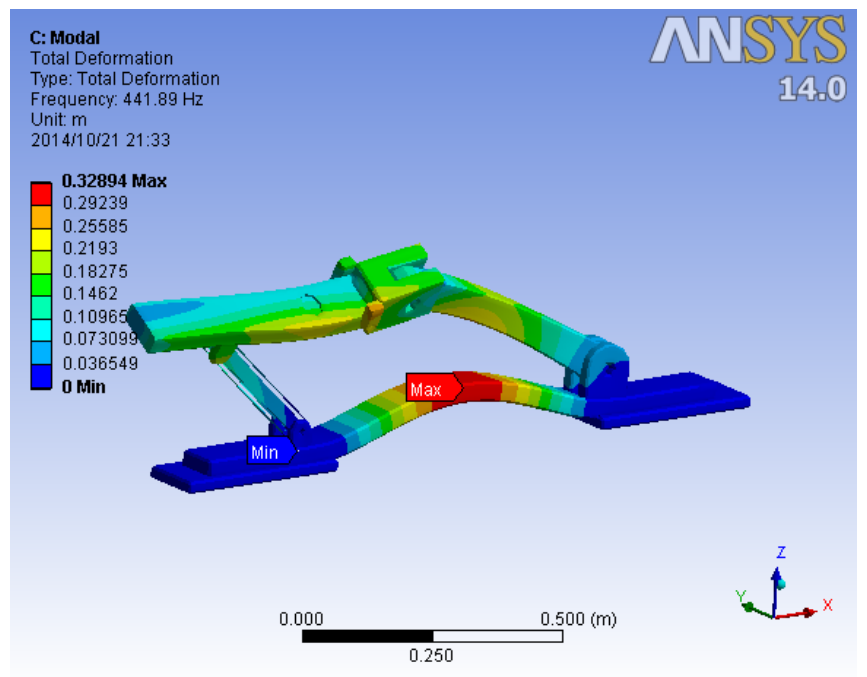

Fig. (7). The $5^{\text {th }}$ order of modality of the base.

\subsection{Determination of Elasto-dynamic Parameters}

Under working condition, the cycling equipment mainly bears time-dependent simple harmonic load and random load. As for random vibration, the statistical sample of time history is converted to power spectral density function (PSD), and analysis is carried out on random vibration based on PSD function to acquire corresponding probability statistics [6]. Assume random load history as $\alpha(t)$, its autocorrelation function can be expressed as follows:

$$
R(\tau)=\lim _{\tau \rightarrow \infty} \frac{1}{T} \int_{0}^{T} \alpha(t) \alpha(t+\tau) d t
$$

Autocorrelation function is a real even function whose frequency on $R(\tau)-\tau$ diagram reflects the frequency components of random load [7-10].

In random vibration analysis, the loads are PSD spectrum which loads on all the constraint positions, as shown in Fig. (8). The diagram shows that the maximum PSD of the structure is $2.2201 \mathrm{e}-010 \mathrm{~m}^{2} / \mathrm{Hz}$ when the frequency is $49.2 \mathrm{~Hz}$.

Periodic excitation was added at revolute joint D according to the force on Point D during operating stroke as provided in Fig. (4). Constraints were added to the base of the cycling equipment as the case may be. Under the excitation of the simple harmonic force, the acceleration-frequency curve of the seat in $\mathrm{Z}$ direction is as shown in Fig. (9). According to the data in the diagram, the response frequency and phase angle of each order are clear. A large acceleration appears at $150 \mathrm{~Hz}$. Therefore, the external periodic excitation around $150 \mathrm{~Hz}$ should be avoided when using the cycling equipment. The maximum phase angle appears at $100 \mathrm{~Hz}$, which is $180^{\circ}$. Fig. (10) and Fig. (11) indicate the strain and stress of the base under the condition that the bottom is fixed. The optimized cycling equipment can meet the requirement of structural strength [11-15].

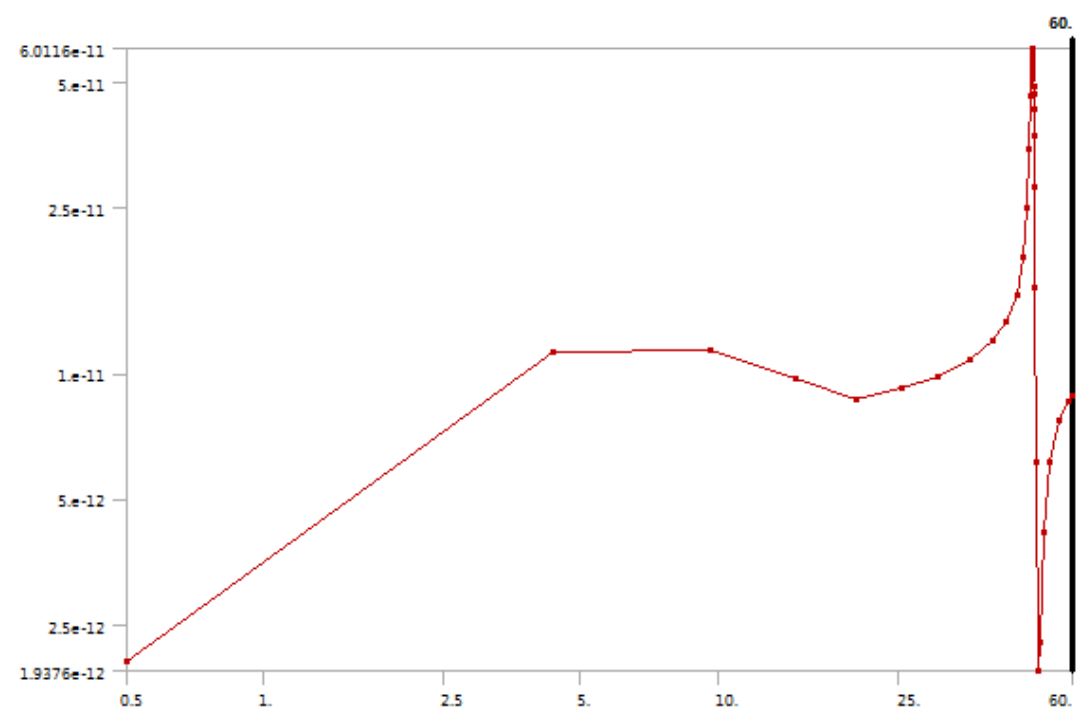

Fig. (8). Diagram of power spectral density. 

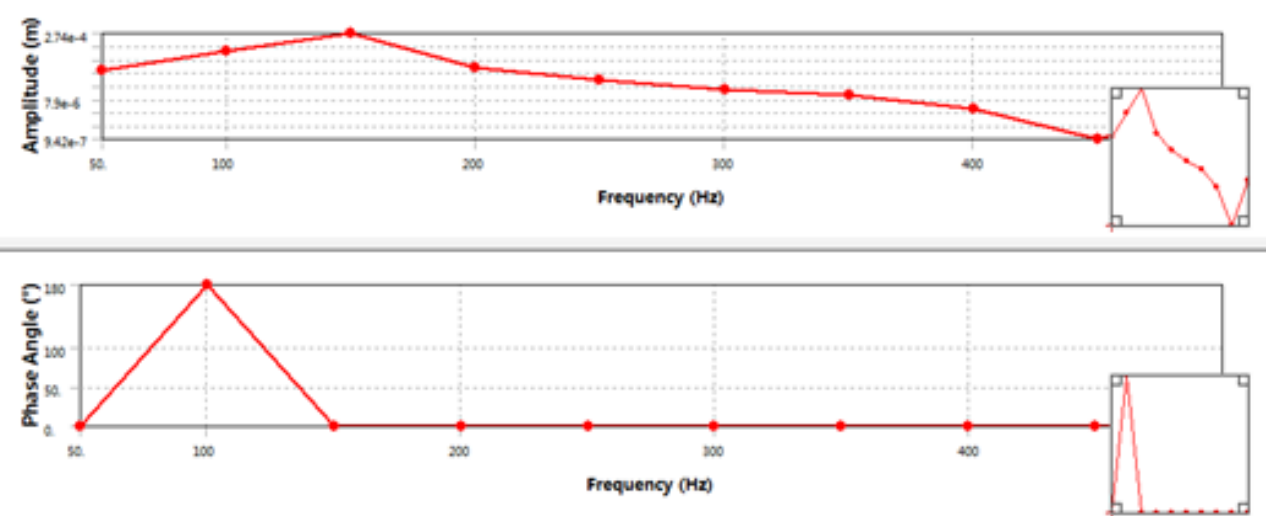

Fig. (9). The response frequencies and phase angles of each order.

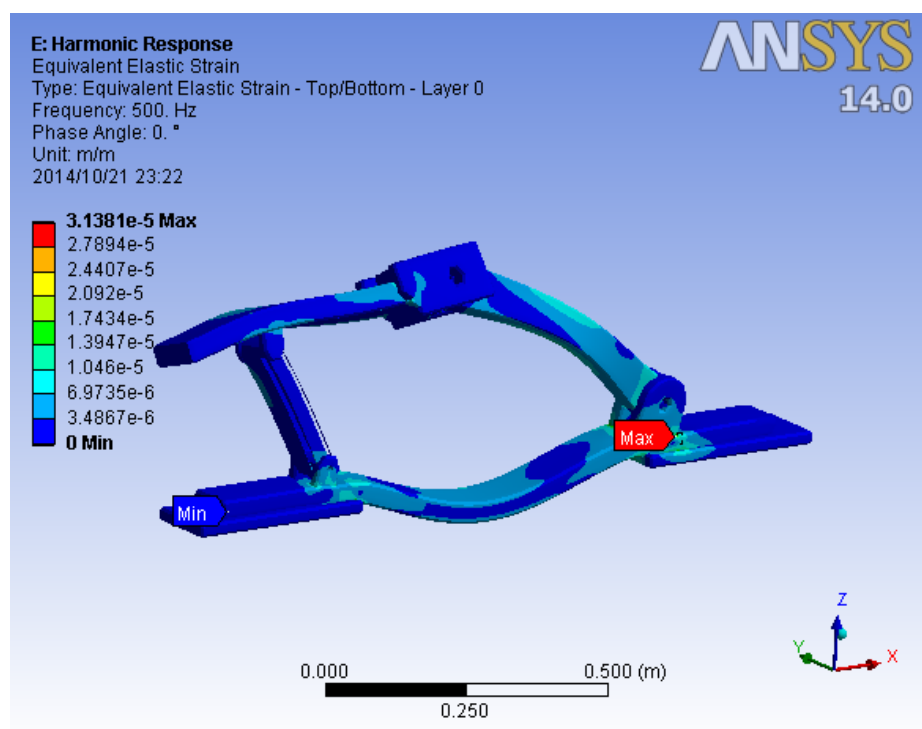

Fig. (10). The nephogram of the strain of the base.

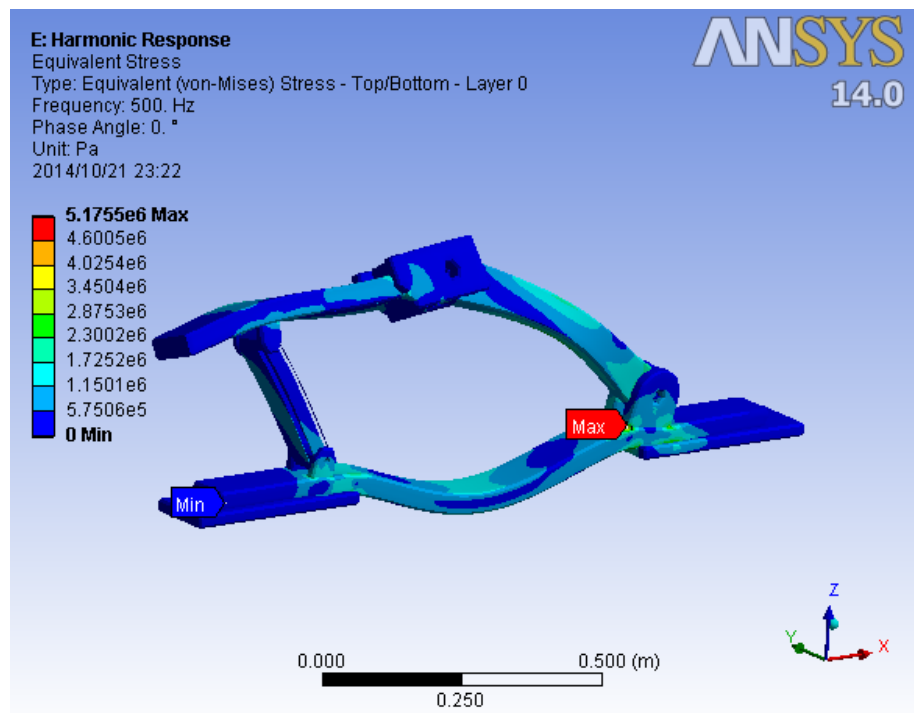

Fig. (11). The nephogram of the stress of the base. 


\section{CONCLUSION}

The many-body dynamic model of the cycling equipment is built using Adams under the condition of meeting ergonomic requirements. Optimization analysis is carried out. To verify the reliability of the optimization results, the structural dynamic characteristics of the optimization are verified and checked by Ansys, through which the dynamic response rules of the main parts of the cycling equipment are acquired. This provides references for improving its dynamic characteristics. A fast and effective method for designing and analyzing cycling equipment is provided in this paper. The method not only takes into account the optimization for parameters, but also ensures the reliability of the product quality. It is a reference method for designing and developing cycling equipment in future.

\section{CONFLICT OF INTEREST}

The author confirms that this article content has no conflict of interest.

\section{ACKNOWLEDGEMENTS}

Declared none.

\section{REFERENCES}

[1] Design and Imitation of the Ridded-Body-Building Machine Based on the Ergonomics, Mechanical Management and Development, vol. 25 , no. 1 , pp. $4-7,2010$.

[2] H. Sun, and W. Ge, Mechanical Principle, Higher Education Press: Beijing, 2006.
[3] X. Hu, and Z. Chen, "The simulation of the plane four links mechanism based on solve function of MATLAB," Mechanical Engineer, vol. 7, pp. 44-45, 2007.

[4] Y. Ding, and Q. Wang, "Ansys ICEM CFD from Introduction to Proficiency," Tsinghua University Press: Beijing, 2012.

[5] Z. Huang, and C. Liu, "ANSYS Workbench 14.0 Super Studying Manual," Posts \& Telecom Press: Beijing, 2013.

[6] S. Yu, L. He, and Q. Guo, "Optimal design of working mechanism in mechanical press based on software ADAMS," Machine Design, vol. 30, no. 2, pp. 25-28, 2013.

[7] J. Wu, D. Dong, B. Yan, and Y. Wang, "Loading scheme design as well as static and dynamic response analysis of hydraulic modular trailers," Machine Design, vol. 31, no. 2, pp. 58-63, 2014.

[8] N. Chen, J. Huang, G. Shi, and L. Liang, "Linear optimize algorithm for motion synthesis of planar four-bar linkage," Machinery Design \&Manufacturer, vol. 8, pp. 21-22, 2007.

[9] K. Liu, C. Liu, J. Lin, X. Ma, and W. Yu, "Harmonic response analysis for bed and column of VMC0540d machine tool," Machinery Design \& Manufacturer, vol. 12, pp. 162-164, 2011.

[10] O. Soegihardjo, and Suhardjono, "Modal and harmonic response analysis: linear-approach simulation to predict the influence of granular stiffeners on dynamic stiffness of box-shaped work piece for increasing stability limit against chatter," International Conference on Mechanical Engineering. Mataram: Trans Tech Publications Ltd., 2014, pp. 501-506.

[11] G. R. Tomlinson, "An analysis of the distortion effects of coulomb damping on the vector plots of lightly damped systems," Journal of Sound and Vibration, vol. 71, no. 3, pp. 443-451, 1980.

[12] S. Chen, R. He, and S. Lu, "Frequency response of suspension with viscidity damping and Coulomb damping", Tractor \& Farm Transporter, vol. 34, no. 4, pp. 34-36, 2007.

[13] F. M. Lewis, "Vibration during acceleration through a critical speed. Trans," ASME, vol. 54, no. 1, pp. 253-261, 1932.

[14] A. Özge, A. Murat, and H. N. Özgüven, "Parametric identification of structural nonlinearities from measured frequency response data," Mechanical Systems and Signal Processing, vol. 25, pp. 11121125, 2011.

[15] MSC/NASTRAN Design Sensitivity and Optimization User's Guide, Version 68, MacNeal-Schwendler Corp., Los Angeles, CA, 1994.

(c) Zhuang Min.; Licensee Bentham Open.

This is an open access article licensed under the terms of the (https://creativecommons.org/licenses/by/4.0/legalcode), which permits unrestricted, non-commercial use, distribution and reproduction in any medium, provided the work is properly cited. 